\title{
Corrigendum
}

\section{Aldehyde dehydrogenase 1A1 expression in breast cancer is associated with stage, triple negativity, and outcome to neoadjuvant chemotherapy}

Thaer Khoury, Foluso O Ademuyiwa, Rameela Chandraseekhar, Marah Jabbour, Albert DeLeo, Soldano Ferrone, Yangyang Wang and Xinhui Wang

Modern Pathology (2012) 25, 917; doi:10.1038/modpathol.2011.203

Correction to: Modern Pathology (2012) 25, 388-397; doi:10.1038/modpathol.2011.172; published online 11 November 2011
In this article, the name of the third author is incorrect. The correct name is Rameela Chandrasekhar. 